\title{
Author Index Vol. 26, 1997
}

\section{(B) $=$ Book Review}

Adelson, P.D. 200 Adler, S.M. 281 Andar, U.B. 17 Anderson, C.A. 255 Ault, C. 83 Avezaat, C.J.J. 269

Bandy, D.J. 144 Barlas, O. 208 Barnett, F.H. 160 Becker, L. 97 Bizzi, J.W.J. 83 Blaser, S.I. 97 Blum, K.S. 33 Boop, F.A. 157 Boydston, W.R. 281 Boyer, R.S. 219 Brockmeyer, D.L. 36 , 168(B) Brodsky, M.C. 157 Brown, K. 25 Bruce, D.A. 83

Castiglia, G. 167(B) Chadduck, W.M. 261,304 Chandramouli, B.A. 214 Chen, K. 144 Cho, B.K. 103 Church, E. 261 Cirak, B. 120 Clyde, B. 200 Colak, A. 120 Connolly, B. 97

Das, B.S. 214 DeBiasi, R. 107 De Leon, G. 277 Deletis, V. 247 Devi, B.I. 214 Dias, M.S. 54, 167(B) Dirks, P. 226 Donahue, D.J. 261,304 Dormans, J. 130 Drake, J.M. 68 Drummond, D. 130 Drury, B.T. 41

Duke, B.J. 107 Duncan, J.D. 144

Elterman, R. 83 Epstein, F.J. 247 
Filley, C.M. 255 Freeman, J.E. 107 Fults, D.W. 36

Garton, H.J.L. 219 Gaskil-LS.J. 115,327 Gilreath, C.L. 281 Gonsalez, S. 296 Goumnerova, L.C. 190 Grant,JJ.A. 8, 56 (B), 57, 166 Gupta,|R. 93

Harkness, W.F.J. 17,288,296 Harpold, T.L. 326 Hayes, T.C. 327 Hayward, R.D. 17,288,296 Hepgül, K. 208 Herman, M. 57 Hes, R. 269 Hoffman, HJ. 68 Holowka, S. 68 Horgan, M.A. 180 Hudgins, P,A. 281

Hudgins, RJJ. 281 Huhn, S. 277 Humphreys, R.P. 68,97 Hunter, S.B. 315 Hwang, P. 68 Hwang, Y.S. 103

Inagaki, T. 185 ízgi, N. 208

Jay, V. 226 Jayakumar, P.N. 214 Jones, B.M. 288,296 Jong, T.H.R. de 269 Jung, S. 226

Kaplan, A.M. 144 Kast, J. 304 Kihç, A. 120 Kịlmç, K. 120 Kim, C.Y. 103 Kim, I.O. 103 Kiriakopoulos, E. 68 Kishore, J. 93 Kleịnschmidt-DeMasters, B.K.

255 Koçak, A. 120 
Kochanek, P.M. 200 Kohli, A. 93 Konan, A. 120 Kothbauer, K. 247 Kurimoto, M. 226 La Marca, F. 8, 57

Langham Gleason, P. 190, 322

Laurent, J.P. 277

Lawson, M.A. 144

Levy, M.L. 326

Liakos, A.M. 236

Linder, S. 83

Logan, W. 68

Luerssen, T.G. 276(B)

Luerssen, T.G. 276(B)

Comb, J.G. 326 McDonald, P.J. 48 McLone, D.G. 1,8,57, 169

225,275,277,312 Madsen, J.R. 190 Magram, G. 236 Malek, A.M. 160 Man waring, K 144 Mapstone, T.B. 25 Marion, D.W. 200 Marlin, A.E. 115 Menick, B.J. 327 Mikulis, D. 68 Morris, R. 281 Moss, S.D. 144 Murakami, M. 226

Nagib, M.G. 2 Nanda, A. 41 Narad,|C. 315 North,|R. 83 Novelli, P.M. 197,322 Nurlu, G. 120

Oakes, W.J. 25 O'Fallon, M.T. 2 Önal, Ç. 208 Orakdögen, M. 208 Otsubo, H. 68

Panigrahi, M.K. 214 Park, J.K》 190 Partington, M.D. 112(B) 
Paz y Geuze, D.H. 269 Petronio, J. 315 Philips, M.F. 130 Piatt, J.H., Jr. 180 Porter-Levy, S. 83 Przybylo, H.J. 312 Przybylo, J. 312

Radkowski, M.A. 53, 277, 312 Raffel, C. 170 Reardon, M.S. 255 Reigel, D.H. 197,322 Reiman, E.M. 144 Rekate, H.L. 113 Rosenthal, A.D. 33 Rutka, J.T. $48,68,226$

Schneider, S.J. 33 Schoenwolf, G.C. 185 Schultz, K.D., Jr. 315 Schut, L. 130 Schwartz, M.S. 160 Scott, R.M. 160,190,219 Shenoy, S.N. 214 Smith, K. 261 Stapleton, S.R. 68 Storrs, B.B. 327 Sutton, L.N. 130

Thompson, D.N.P. 288,296 Thompson, G. 36 Tomita, T. 8 Townsend, J.J. 219 Tsugu, A. 226 Tyson,,R.W. 107

Ünal, F. 208

Walker,M.L. 36, 185,219 Wang, K, C, 103 Wilkening, G.N. 255 Winston, K.R. 107 Wisniewski, S.R. 200 Wodrich, D.L. 144

Yonas, H. 200

Yunis, E. 322

\section{KAIVGER}

Fax + 4l 613061234 E-Mail karger@karger.ch www. karger.|com 
